\title{
thebmj
}

Letters Abdominal aortic aneurysm

\section{Comparing studies for cost effectiveness of screening}

BMJ 2009; 339 doi: http://dx.doi.org/10.1136/bmj.b3044 (Published 28 July 2009) Cite this as: BMJ 2009;339:b3044

\author{
Simon Thompson, director ${ }^{1}$, Lois Kim, lecturer2, Lu Gao, statistician 1 \\ ${ }^{1}$ MRC Biostatistics Unit, Institute of Public Health, Cambridge CB2 OSR \\ ${ }^{2}$ London School of Hygiene and Tropical Medicine, London WC1E 7HT
}

simon.thompson@mrc-bsu.cam.ac.uk

Ehlers and colleagues conclude from their long term modelling study that screening men aged 65 for abdominal aortic aneurysm is not cost effective. 1 This conclusion conflicts with that of the 10 year follow-up of the randomised Multicentre Aneurysm Screening Study (MASS), 2 our detailed long term modelling based on individual patient data in MASS, 34 and other recent modelling studies.

To try to understand the reasons for the difference, we substituted the unit costs and parameter estimates provided by Ehlers into our model based on MASS. Although the cost per quality adjusted life year (QALY) increased from our original estimate of $£ 3000$ to around $£ 6000$, it does not begin to approach their figure of $£ 43000$. So there must be other explanations, such as the structure or assumptions of their model, which cannot be investigated without further information from the authors.5

Their cost effectiveness estimate is implausible. From observed data in MASS, we showed that cost effectiveness after 10 years was $£ 9400$ per QALY.2 Cost effectiveness will improve when considered over the longer term, since costs are generally up front while benefit accrues over time. But Ehlers and colleagues' estimate of lifetime cost effectiveness is worse than that which we have already observed after 10 years. Moreover, they present the modelled number of deaths related to abdominal aortic aneurysm that accrue as a screening programme is launched, and claim that a net reduction is not reached until after nine years. Rerunning this analysis based on the data observed in MASS shows that benefit is seen after two years.

Ehlers and colleagues' hypothetical modelling does not agree with the data observed in the MASS trial, which provides most of the worldwide randomised evidence. If one had to choose the basis on which to make policy decisions, real data are surely preferred.

\section{Notes}


Cite this as: $B M J$ 2009;339:b3044

\section{Footnotes}

- Competing interests: None declared.

\section{References}

1. Ehlers L, Overvad K, Sorensen J, Chistensen S, Bech M, Kjolby M. Analysis of cost effectiveness of screening Danish men aged 65 for abdominal aortic aneurysm. BMJ2009;338:b2243. (24 June.)

2. Thompson SG, Ashton HA, Gao L, Scott RAP. Screening men for abdominal aortic aneurysm: 10 year mortality and cost effectiveness results from the randomised Multicentre Aneurysm Screening Study. BMJ2009;338:b2307. (24 June.)

3. Kim LG, Thompson SG, Briggs AH, Buxton MJ, Campbell HE. How cost-effective is screening for abdominal aortic aneurysms? Journal of Medical Screening 2007;14:46-52.

4. Kim LG, Thompson SG, Buxton MJ, Briggs AH, Campbell HE. A Markov model for long-term cost-effectiveness modelling of screening for abdominal aortic aneurysms. MRC Biostatistics Unit Technical Report2005/2. Available at: www.mrc-bsu.cam.ac.uk

5. Buxton MJ. Screening for abdominal aortic aneurysm. BMJ2009;338:b2185. (24 June.) 